\title{
Intelligent looks
}

\section{Graham A. Dunn}

Journal of Computer-Assisted Microscopy. Editor-in-chief John C. Russ. Plenum. 4/yr. US and Canada \$115; elsewhere $\$ 135$.

THERE is hardly a human endeavour that is not computer-assisted these days and so perhaps the title of this new journal is intended to warn off 'computerphobes' rather than to suggest a highly specialized area of microscopy. Computers are certainly playing a central part in the revolution that is happening in microscopy: sophisticated image processing is helping to erode the old 'fundamental' limits on the type of information that can be gathered optically; many innovations at the forefront of microscope technology are entirely dependent on digital processing and even routine data from microscopes are now rarely interpreted or quantified without some form of computation being used.

The main role of computers in this revolution has so far been rather mundane. Most of the image processing and analysis is commonplace and the computer control of microscope functions is still largely rudimentary. But computers are already proving to be an essential adjunct to certain new types of microscopy and must soon be regarded as part of the microscope, as indispensible as the optics. The enormous quantity of information that can be generated by scanning confocal microscopy, of living material, for example, can only be stored and adequately interpreted through digital processing. A vision of the future in which we can effortlessly explore the dynamic four-dimensional structure of a living, developing embryo, changing our direction of view at will and moving back and forth in both time and space, is probably now more contingent on progress in software and in digital processing than on progress in optical technology.

This new journal is timely because microscopists must now acquire expertise in computing to avoid becoming slaves to manufacturers' hidden software. No analysis of scientific data should be acceptable for publication if the investigator is ignorant of the algorithms used. The first few issues have done well to bypass the more routine aspects of image processing and storage and have concentrated on computer applications that do not normally appear in books on the latest microscope techniques. The main emphasis seems to be on data reduction and analysis and there is a surprising wealth of information on what may be loosely described as metrology and stereometry: harmonic shape analysis, surface curvature analysis, adjacency measures in tessellations, and so on. These are obviously the main areas of interest of the editor-in-chief, who has already contributed no less than 12 mathematically sophisticated articles. Taken as a whole, the articles cover a ragbag of topics from correction of piezoelectric creep in scanning tunnelling microscopy to goniospectrophotometry

\section{Thinking big}

\section{R. J. P. Williams}

Journal of Biomolecular NMR. Editor-inchief Kurt Wüthrich. ESCOM. 6/yr. Dfl. $600, \$ 310$ (institutional); Dfl. 190, $\$ 95$ (personal).

A JOURNAL of this title has to be largely concerned with biological polymers because NMR characterization of smaller molecules, whether they be biological, organic or inorganic, is common ground for many chemistry journals and is fairly routine. No new organic molecule, even fulvarenes, can now be described in an acceptable manner without an NMR spectrum. So what is special about largemolecule NMR? It is very difficult to analyse with great confidence NMR spectra of molecules that exceed a relative molecular mass of about 4,000 (4K) and that are not rigid, and the problem becomes horrendous for molecules of $50 \mathrm{~K}$ or more. This journal is concerned with these problems for large biological polymers.

Although NMR can give a good picture of the structure of the more rigid elements of regular secondary features of, for example, a protein or a DNA fragment in solution, we do not really know how good the pictures are of irregular strands or side chains. The problem has two parts: can the methods used define rigid structure, if any, accurately; and can any nonrigid structure be determined at all in solution? The papers in the first issue reveal these problems. There are three papers on assignment procedures, that is operational problems before structural analysis, three on structural study and three on dynamics. The trend is to ever-more sophistication in assignment technique and in theory. Clearly it is useful in one sense to discuss these problems and the technical developments all in one place, but could not the problems have been managed under the Journal of Magnetic Resonance? Many libraries will not subscribe to yet another spectroscopy journal, especially with the increase in the size of existing journals. of meat. The only consistent theme that I could detect is that the articles are sure to be enjoyed by computer buffs and by microscope innovators alike. One of the manufacturers of scientific equipment assures us that the age of really intelligent microscopes has arrived; perhaps the new journal will provide a forum for them to communicate with each other. $\square$

Graham A. Dunn is in the Medical Research Council Muscle and Cell Motility Unit, King's College London, 26-29 Drury Lane, London WC2B 5RL, UK.

Judging from the quality of the editorial board and the first issue, good papers will be generated for at least a short while. But will the journal last over a longer period? Will it publish NMR studies of biomolecules that have been analysed by crystal-structure studies? Crystallographers often criticize 'structures' deduced by NMR methods while forgetting the complementary nature of the knowledge gained, especially on chemical interactions and dynamics in solution. And is a separate journal on NMR spectroscopy of biopolymers really warranted for the study of biological structures? Would not existing combinations of journals on NMR on the one hand and biological structures on the other serve better? And at this price, the journal is very discouraging except for rich institutes.

R. J. P. Williams is in the Inorganic Chemistry Laboratory, University of Oxford, South Parks Road, Oxford OX1 3QR, UK

\section{Ringing the changes}

\section{Stanley Roberts}

Polycyclic Aromatic Compounds. Editorin-chief M. Zander. Gordon and Breach. 4/yr. UK Dfl. 1287, rest of Europe ECU 557, US and Canada $\$ 595$, elsewhere SwFr. 1170 (corporate); Europe Dfl. 825 , US and Canada $\$ 375$, elsewhere SwFr. 750 (academic library); UK 1108 , rest of Europe ECU 179, US and Canada $\$ 168$, elsewhere SwFr. 384 (personal).

THE aim of this new journal is to collate information from scientists working with polycyclic aromatic compounds in biology, physics and chemistry laboratories. Short papers, full-length articles and reviews are welcomed.

Polycyclic aromatic compounds are undoubtedly important - for example some products of their oxidation are carcinogenic. Nonetheless, it is doubtful whether they justify a journal all to themselves, especially considering the current huge proliferation of journals. 